\title{
The Importance of Serology Examination in Diagnosis of Multiple Ulcers in Oral Cavity (Serial Cases)
}

\author{
Rolis Anggi Wuriyanti \\ Oral Medicine Resident \\ Faculty of Dentistry, Universitas Indonesia \\ Jakarta, Indonesia \\ roliswuri_13@yahoo.com \\ Gus Permana Subita \\ Department of Oral Medicine \\ Faculty of Dentistry, Universitas Indonesia \\ Jakarta, Indonesia \\ guspermana11@gmail.com
}

\author{
Anandina Irmagita Soegyanto \\ Department of Oral Medicine \\ Faculty of Dentistry, Universitas Indonesia \\ Jakarta, Indonesia \\ a.irmagita@gmail.com \\ Harum Sasanti Yudoyono Nugroho \\ Department of Oral Medicine \\ Faculty of Dentistry, Universitas Indonesia \\ Jakarta, Indonesia \\ harumsasanti@gmail.com
}

\begin{abstract}
Recurrent Aphthous Stomatitis (RAS) and Recurrent Intraoral Herpes (RIH) are the most common oral lesions found in dental practice. The diagnosis can be made based on patient's history and clinical examination. Due to similarity of size, shape, number, and location of these ulcers, however, it may be difficult to distinguish both of it. Serology examination of HSV-1 and HSV-2 antibody is often used to establish the diagnosis. Case: Two male patients, 26 and 43 years old, came to Department of Oral Medicine with complaints of sore mouth that were not preceded by fever. From clinical examination, multiple ulcers were found in keratinized and non keratinized mucosa in sizes of $0.5-3 \mathrm{~mm}$. Serology examination showed negative result for both anti HSV in first patient and positive result for anti HSV-1 IgG in second patient. As a result, the definitive diagnosis for first patient was Herpetiform Aphthous Stomatitis and for second one was RIH. Chlorhexidine Gluconate (12\%) mouth rinse and multivitamin were prescribed for first patient and $200 \mathrm{mg}$ Acyclovir tablets (5 times a day for two weeks); $100 \mathrm{mg}$ Doxycycline mouth rinse and multivitamin were administered for second patient. In conclusion, Serology examination is necessary to establish diagnosis of disease with similar intraoral multiple ulcers pattern and to help the dental practitioner in developing proper management for the patient.
\end{abstract}

Keywords-RAS, RIH, herpes, serology

\section{INTRODUCTION}

Multiple ulcers are the most common cases found in daily dental practice. Some are easily recognizable only by clinical examination, but others may be difficult to be identified without additional examinations due to their similarities in history and characteristics.

Recurrent Aphthous Stomatitis (RAS) and Recurrent Intraoral Herpes (RIH) are frequently misinterpreted. The ambiguity in diagnosing these lesions is reasonable as both have similar clinical appearances, but differ in nature. RIH results from viral infection, while RAS has unknown etiology.

RAS is a chronic inflammatory disease forming single or multiple painful ulcers, which are round or oval in shape, yellowish white in colour, with halo erythema in surrounding area. The ulcer is preceded by burning sensation that lasts about 2-48 hours in nonkeratinous mucosa, e.g. buccal, or labial mucosa, floor of mouth, or tongue. The etiology of RAS is unclear, but there are several predisposing and triggering factors contributing to its occurrences Predisposing factors include genetic, food allergies, vitamin deficiency, and immune system alterations, and stress, while triggering factors include local, physical or chemicals trauma, infection and smoking habit. There are three types of RAS, namely minor, major, and herpetiform. Minor aphthous stomatitis is the most common type with characteristics of 5-10 $\mathrm{mm}$ in size, 1-5 lesions in numbers, and 10-14 days recovery without production of scar tissue. Major aphthous stomatitis is characterized with 1-10 lesions with size of more than $10 \mathrm{~mm}$ and 2 weeks to 1 month recovery period with scar tissue. On the other hand, herpetiform aphthous stomatitis is the least and rarest type, characterized with 10-100 lesions measuring less than $5 \mathrm{~mm}$ which could coalesce to form larger ulcers. The duration of healing period is about 10-14 days with higher pain severity than other two types $[1,2,3]$.

RIH is a reactivation of latent herpes virus in trigeminal ganglia. This lesion is triggered by decreased immune system that may be due to stress, menstruation, trauma, immunosuppressant, or chemotherapy cycle. RIH is usually preceded by prodromal symptoms, e.g. fever, discomfort, anorexia, lymphadenopathy, sore throat, itching, and tingling before the ulcer appears [4]. This period indicates that the replication of virus is occurring in the sensory nerve ending for several hours 
to days. The reddish maculopapular lesions will then appear and rapidly turn into white grey vesicles that measures less than $1 \mathrm{~mm}$. The vesicles multiply and rupture forming ulcers with sizes of approximately 1.5 $\mathrm{cm}$, lasting for 2 to 4 days [5]. In oral cavity, the ulcer can appear in either non-keratinized or keratinized mucosa, such as hard palate, attached gingival, alveolar ridges, and vermilion borders of the lips [4,6,7] with irregular border and halo erythema [7]. Crusting does not occur [8]. During this period, it is very contagious because HSV-1 can repeatedly go out from saliva [5].

The diagnosis RAS is basically established based on clinical examination, unless the lesions are triggered by underlying systemic disease. Unlike RAS, RIH requires more additional methods in its diagnosis, especially to identify antigen, antibody, and tissue reaction of herpes virus infection, such as Tzank test, Polymerase Chain Reaction (PCR), direct immunofluorescence antibody and serologic test $[6,7]$. The most commonly used is serologic examination [6]. This test is useful either in symptomatic patients by giving positive results or in asymptomatic patients by exploring whether the infections is acute, chronic, or exacerbating $[6,9]$.

This case report is discussing about two cases of multiple ulcers, herpetiform aphthous stomatitis and recurrent intraoral herpes with similarities in characteristics and location, thus requires serological tests to establish the definitive diagnosis. Both patients are from Oral Medicine Clinic, Dental Hospital Faculty of Dentistry, Universitas Indonesia.

The purpose of this paper is to show the importance of serologic examination as an additional tool to identify multiple ulcers with similar clinical characteristic in oral cavity, so that appropriate treatment plan can be developed.

\section{CASE REPORTS}

\section{A. Case I}

A 25-year-old male patient, working as health practitioner in public hospital, came with a chief complaint of multiple ulcers throughout oral cavity since 4 days ago. He has previous similar experience two months ago, with lesions found only on right buccal mucosa and floor of mouth which healed within 10 days. He didn't notice why these ulcers appeared. He tried rinsing with OTC mouthwash and consuming multivitamin. The ulcers currently appeared in more numbers, starting with vesicles, burning sensation, and lymphadenopathies that was thought as a malignancy. Patient admitted to being under stress and was afraid that this disease might be related to his works which was prone to infection of HIV. He had a history of 10year smoking and ossifying fibroma surgery 2 years ago in right maxilla but post operation area was not completely closed.

In extraoral examination, it was found that there were lymphadenopathies which were palpable in consistency but painless on both sides. In intraoral examination, a papule sized $0.5-1 \mathrm{~mm}$ with same color as its surrounding area were found in region of 12 and
23 respectively. Multiple ulcers that had regular borders, yellowish white in base, and surrounded by halo erythema were found in non-keratinized and keratinized mucosa in size of $0.5-2 \mathrm{~mm}$, with some coalescing to form larger lesion of up to $4 \mathrm{~mm}$ (Figure 1). Oral hygiene was poor, indicated by the presence of calculus, debris, and stain throughout oral cavity.

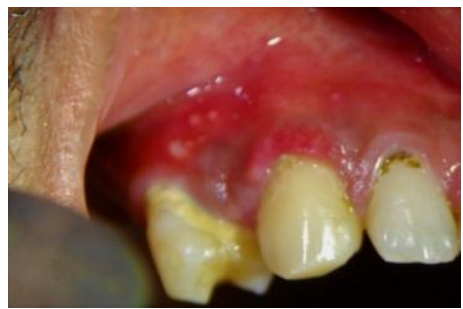

Figure 1. Multiple ulcers in gingiva.

\section{B. Case II}

A 43-year-old male patient came with a chief complaint of tingling, burning, and painful sensation throughout his oral cavity. He first experienced this 2 month ago which later persisted to the appearance of multiple ulcers in upper and lower lips. This ulcer was never completely healed so the patient came to hospital 3 weeks ago to obtain further therapy and was given prescription of antibiotic, antiinflammation agent, multivitamin, mouthwash, and oral cream. He also used OTC mouthwash to fasten healing process to no effect. He revealed that prior to the occurrence of ulcers; he had physical stress due to overworking, disturbed rest, and infrequent eating. In the previous three weeks, he had experience of malaise but no fever. He tried to have three meals a day by consuming more fruit, vegetables, and high protein foods. History of systemic disease was neglected. However, he had a history of smoking for 30 years ago but had stopped 3 weeks ago.

Extraoral examination revealed lymphadenopathy on both sides which is palpable in consistency but painless. Intraoral examination revealed multiple irregular and coalesced ulcers, sized 1-8 mm, with yellowish white base, and located in buccal mucosa, labial mucosa, lateral and ventral of tongue, and soft palate, and pharynx (Figure 2). The oral hygiene was poor, indicated by the presence of calculus, debris, stain, and necrotic teeth.

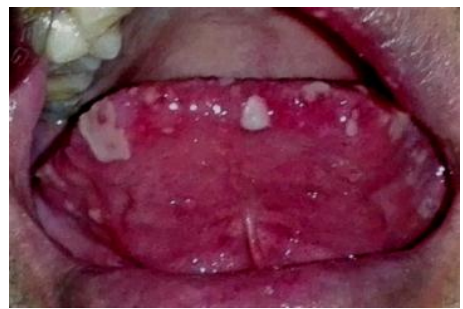

Figure 2. Multiple irregular and coalesced ulcers

\section{CASE MANAGEMENT}

A. Case I

On the first visit, patients were given $0.12 \%$ chlorhexidine gluconate and multivitamin and recommended to perform serology laboratory tests of 
anti HSV-1 \& 2 IgG and IgM level, complete hematologic examination and to come for control 1 week later. Patients are also instructed to eliminate the focus infection and to maintain oral hygiene.

One week later, on the second visit, the lesion in the oral cavity had improved. Serological examination revealed negative results and diagnosis of RAS herpetiform was established. Based on the diagnosis, the patient was given an explanation of the RAS disease that can appear any time with few or many lesions based on the trigger factors experienced by patients. In addition, patients are advised to improve the cleanliness of the oral cavity by cleaning the tartar.

\section{B. Case II}

On the first visit, the patient was given prescription of $100 \mathrm{mg}$ doxycycline mouthwash for 3 times a day and multivitamin and suggested to perform serology test of anti HSV-1 and HSV-2 and complete hematological examination.

On the second visit, four days after the first visit, the patient came with a positive laboratory result of the anti IgG HSV-1. Thus, the diagnosis of recurrent simplex herpes or RIH infections was established. The prodromal symptom of itchiness on the tongue and lips and soreness during swallowing were confirmed by patient. Clinical examination results showed widening of palatal ulcers (Figure 3), and occurrence of new lesion in the labial mucosa was found (Figure 4).

Based on the results of serological tests and the presence of prodromal symptoms, patient was given $200 \mathrm{mg}$ of additional acyclovir therapy, 5 times daily for 10 days, considering possibility of a new lesion and preventing the risk of transmission.

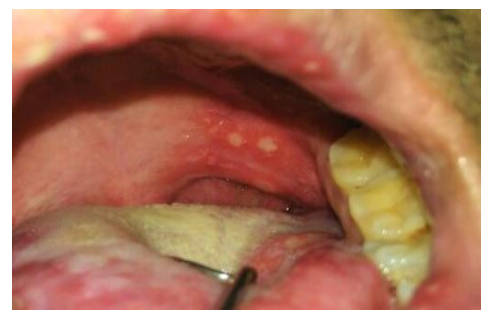

Figure 3. Widening of palatal ulcers.

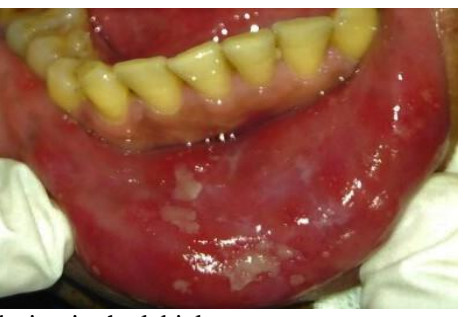

Figure 4. New lesion in the labial mucosa.

\section{DISCUSSION}

Clinical appearance and location of both lesions is similar to the characteristics of herpes simplex infection, particularly involving attached gingiva in the first case and pharyngotonsil area as predilection of recurrent herpes infection in the second case. Laboratory examination is important to establish diagnosis for both cases so that a more accurate treatment plan can be developed, especially in giving prescription of antiviral therapy and instructions on the prevention of recurrences and transmissions.

Definitive criteria in diagnosing herpes simplex infection are usually by isolation and culture of virus taken from fresh vesicles. This process requires almost two weeks to be evaluated with Tzank smear, PCR, or immunofluorescent antibody. As obtaining fresh vesicles from mouth is difficult due to its fragility, serology test becomes more preferable [8]. This test uses enzyme-linked immunosorbent assay (ELISA) or immunoblot to assess antibody HSV-1 and HSV-2, IgG and $\operatorname{IgM}$ to achieve $97-100 \%$ in specification result and $98 \%$ in sensitivity. This method also has faster time of seroconversion compared to Western Blot method [9].

Another benefit of serology test is its ability to detect viral infection in early evaluation. It is used if a more complex isolation procedure (EBV, HHV-6) and animal inoculation are needed. It is also used if the virus may not be seen when the clinical symptoms develop. For some viral infection, e.g. measles, rubella, mumps, rubella, serology test is faster and cheaper than culture examination. Besides, it can be used as diagnostic confirmation of $\mathrm{HCV}$ and $\mathrm{HIV}$ in early detection within 8-14 days [10].

Serology test is used to measure immune status and viral infection history of a person $[5,10]$. New occurrence or recurrence of infection might be indicated by the appearance of specific IgM during acute period or the elevation of specific IgG between acute and healing phase. Specific $\operatorname{IgM}$ is usually found in bloodstream in first week of primary infection and cannot be detected within 1-3 months, while $\mathrm{IgG}$ is usually produced in 1-2 weeks after primary infection, reaching a peak level during 4-8 weeks, gradually decreasing but could still be detected without time limit. Specification of HSV is being repaired by choosing antibody to specific antigen (glycoprotein G) where secondary immune response occurs after viral reinfection or reactivation to produce different serology profile that is the temporary appearance of IgM in low amount and fast increase of IgG. All this is influenced by infected virus, location, and host immune status [10].

In conclusion, serologic examination is necessary to establish the definitive diagnosis when subjective and objective examination show similarities, especially in multiple ulcer lesions. In addition, appropriate interpretation of laboratory results may also be an important consideration before prescription of antiviral therapy, especially in patient with high risk of recurrence to reduce the transmission risk.

\section{REFERENCES}

[1] A.M. Kumar, V. Ananthakrisnan, J. Goturu, "Etiology and pathophysiology of recurrent aphthous stomatitis: A review," Int. J. Cur. Res. Rev., vol. 06, pp. 16-22, May 2014.

[2] S.O. Akintoye, M.S. Greenberg, "Recurrent aphthous stomatitis," Dent. Clin. N. Am., vol. 49, pp. 31-47, 2005.

[3] Z. Ślebioda, E. Szponar, A. Kowalska, "Etiopathogenesis of Recurrent Aphthous Stomatitis and the Role of Immunologic 
Aspects: Literature Review," Arc. Immunol. Ther. Exp., vol. 62, pp. 205-215, 2014.

[4] J.R. Gurenlian, "Differentiating herpes simplex virus and recurrent aphthous ulserations," DHDx and Oral Medicine, pp. 30-34, February 2003.

[5] K. Gutierrez, B. Pinsky, A.M. Arvin, "Textbook of Pediatric Infectious Diseases," $7^{\text {th }}$ ed., Elsevier, 2014, 1933-1968.

[6] S. Bhateja, G. Arora, S.K. Mastud, "Recurrent intraoral herpes (RIH) infection - A Case Report,” Biomed. J. Sci. \& Tech. Res., vol. 1, pp. 1-3, August 2017.

[7] T.S.I. Tilliss, J. McDowell, "Differential diagnosis: Is it herpes or aphthous?," J. Contemp. Dent. Pract., vol. 3, pp. 1-15, February 2002

[8] A. Singh, J. Preaiksitis, B. Romanowski, "The laboratory diagnosis of herpes simplex virus infections," Can. J. Infect. Dis. Med. Microbiol., vol. 16, pp. 92-98, 2005.

[9] L.J. Mazur, M. Costello, "Clinical Diagnosis and Management by Laboratory Methods," 23th ed. Elsevier/Saunders: Philadelphia, 2017, 1072-1098. 